\title{
Design of steering system for a small Formula type vehicle using tire data and slip angles
}

\author{
Sadjyot Biswal ${ }^{1}$, Aravind Prasanth ${ }^{1}$, Dr. R Udayakumar ${ }^{2}$, Dr. M. N. Sankaram ${ }^{2}$, Dhruvil Patel ${ }^{1}$ \\ ${ }^{1}$ Automotive, Mechanical Department, BITS Pilani Dubai Campus, DIAC, 345055 \\ ${ }_{2}^{2}$ Mechanical Department, BITS Pilani Dubai Campus, DIAC, 345055
}

\begin{abstract}
The focus of this paper would be on the design of steering system for a small formula race type vehicle. The design would consist of determining steering geometry with the use of tire models and optimizing the deign to have the best possible design. Ackermann Geometry, Anti Ackermann, parallel steer will be discussed and the ways to obtain the desired geometries will also be focussed on. The effect of steering on other parameters like roll centre will also be discussed. The importance of reducing bump steer and the methods of doing it will also be discussed.
\end{abstract}

\section{Introduction}

The focus of this paper is to understand dynamics of vehicle related to design of steering geometry and how to design a steering geometry as per track requirement and use of tire data. The paper will focus both on static and dynamic design of the steering system and will take into consideration the parameters to be kept in mind while designing a fast responding system with enough steering feel. The paper will present a step by step analysis of the design phase and how a kinematic steering software can be used to determine correct geometry.

\section{Requirements of steering system}

The steering system needs to be such that the car can cover all turns on the track and also give accurate steering feel to the driver. The steering effort required by the driver should not be too much that it makes it uncomfortable and at the same time maintains enough steering feel.

\section{Design}

The most important concepts for designing a race car is to keep the tire operating at peak lateral force. The peak lateral force of the tire is a property of the tire and depends on the inclination angle and normal load on the tire. To determine the peak lateral force at a particular slip angle, at a normal load and slip angle is determined by the tire data which can be purchased from FSAE TTC. This will provide some knowledge as to what type of steering geometry is suited for the tires.

\subsection{Three types of steering geometry}

Ackermann: In Ackermann geometry, the inner tire in a corner turns more than the outer tire with the same input steering angle.

Anti-Ackermann: In Anti Ackermann geometry, the inner tire does not turn as much as the outer tire with the same input steering angle.

Parallel Steer: In parallel steer both the wheels will turn by the same amount with same input steering angle.

According to the weight transfers in the corners, the different peak lateral forces and the slip angles for it can be noted according to the Lateral force vs Slip angle graph. Then depending on it the steering geometry which is preferred by the tires to operate at peak lateral force can be found out

The weight transfer can be found out using the below Formulae.

$$
\begin{gathered}
M_{\text {roll }}=A_{y} m h \\
\theta_{\text {roll }}=\frac{M_{\text {roll }}}{K_{f}+K_{r}} \\
F_{z r}=0.5 \rho m g+\frac{\theta_{\text {roll }} K_{f}}{t_{f}} \\
F_{z l}=0.5 \rho m g-\frac{\theta_{\text {roll }} K_{f}}{t_{f}} \\
A_{y}=\frac{F_{y l}+F_{y r}}{\rho m}
\end{gathered}
$$

Where

-Ay is the lateral acceleration

-Mroll is the roll moment

- $\theta$ roll is the roll angle

$\bullet$ Fzr and Fzl are the right and left tire vertical loads 
-Fyr and Fyl are the right and left tire lateral forces

- $g$ is the acceleration due to gravity

The normal load after weight transfers have been found out as $1431 \mathrm{lb}$ and $2601 \mathrm{lb}$ respectively.

Now once the weight transfer has been found out, we need to find the inclination angle of the tires for the cornering as well.

This can be found out using the below formula:

$$
\gamma_{r}=\gamma_{r 0}+\lambda\left(1-\cos \delta_{r}\right)-v \sin \delta_{r}+\theta_{\text {roll }} C
$$$$
\gamma_{l}=-\gamma_{r 0}+\lambda\left(1-\cos \delta_{l}\right)-v \sin \delta_{l}+\theta_{\text {roll }} C
$$

Now once the inclination angle and normal load on both the front tires have been found out, we can use the tire data and use it to find out the slip angles at which peak lateral force can be achieved. As the tire data is a raw data we need a tire analysis software to get the $F_{y}$ vs SA graphs. Optimum $\mathrm{T}$ has been used for the following case.

We notice from the graph that the lateral force peaks out at a particular slip angle and then falls down. The graph points can be exported into an excel sheet and the peak lateral force can be found out and noted.

For the particular set of tires, the normal loads and inclination angles calculated are:

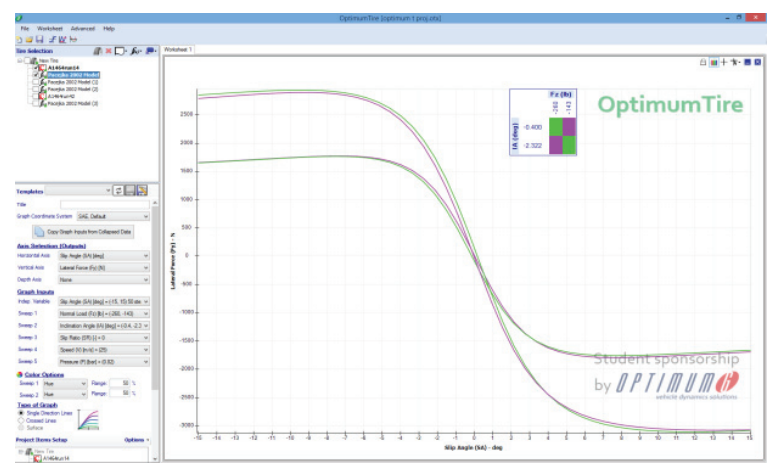

Figure 1. orce vs Slip angle

Fz Outer $=-260 \mathrm{lb}$

Fz Inner=-143 lb

Inclination Angle Outer: - 0.4

Inclination Angle Inner: - 2.322

The corresponding slip angle values for the tires to operate at peak lateral force for the above-mentioned set of values are:

11.93 for the outer tire and 7.65 for inner tire.

The above results show that these particular set of tires prefer anti Ackermann steering geometry as the outer tire needs more slip angle and hence more steering input to obtain peak lateral force values.

\section{Tire drag}

For a formula student car and other low downforce formula type cars which run on tracks with tight corners, one more factor comes into play which determines what kind of steering geometry that should be used called tire drag.
Tyre drag is a effect of the lateral force often called as lateral force induced drag. It is a force in the opposite direction of vehicle movement analogous to aerodynamic drag but created due to the tire. Higher the slip angle at which the car operates more the drag produced by the tire. This tire drag has a huge effect on cornering of a vehicle.

Whenever a vehicle is needed to traverse a corner, it needs yaw moment. For a tight turn like a hairpin where a large lateral acceleration is needed in a small time, tire drag has a very negative impact on the turning of the car.

From the figure above it can be seen that the tire drag on the inner tire helps to traverse the corner but he drag on the outer side produces a moment out of the corner. If Anti Ackermann is preferred then the outer tire would have a large tire drag and hence the vehicle wont be able to take tight corners as found in Formula student circuits.

Therefore, based on one's track and requirement a particular steering geometry must be chosen. Due to the needs of FSAE track being tight corners Ackermann steering geometry is considered to be more suitable.

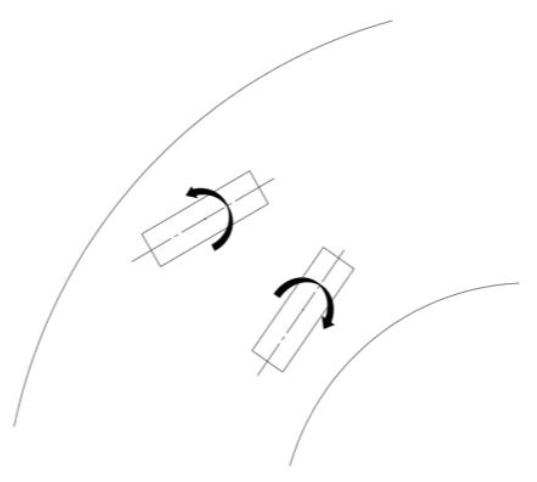

Figure 2. Tire Drag

\section{How to determine steering curve}

Now to determine the steering curve there were two things to be seen, the tires wanted a anti Ackermann geometry whereas considering the tire drag Ackermann geometry would be more preferred. Therefore, a small Ackermann geometry was preferred so as to reduce the effects of tire drag and still not sacrifice a lot of the tire's peak lateral force.

The steering curve is basically a graph between the Tyre Inside-Tire Outside angle vs Ackermann Angle. To determine the difference between Inside and Outside angles the following set of formulas have been used.

$$
\alpha_{r}^{G}=\frac{l}{R+\frac{t}{2}}+\beta
$$

$$
\begin{gathered}
\alpha_{l}^{G}=\frac{l}{R-\frac{t}{2}}+\beta \\
\delta_{r}=\alpha_{r}^{G}-\alpha_{r}^{i}
\end{gathered}
$$




$$
\delta_{l}=\alpha_{l}^{G}-\alpha_{l}^{i}
$$

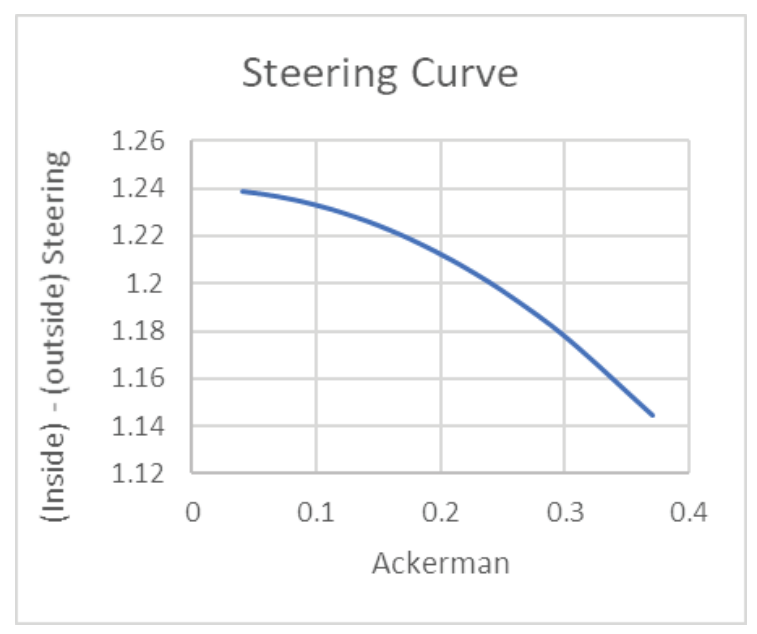

Figure 3. Steering Curve

The track radius can be determined form the Optimum L software which has inbuilt track co-ordinates of FSAE track. From the software, it can be seen that majority of the car travels at corners of radius and maximum radius required to go across is $25 \mathrm{~m}$ and minimum is $4.5 \mathrm{~m}$

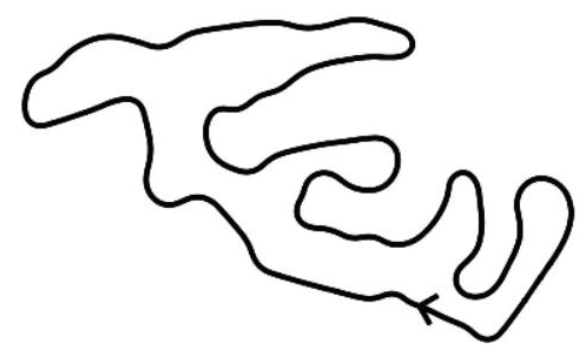

Figure 4. FSAE Germany Endurance track

Now it needs to be seen if the particular steering geometry can cover the lowest possible radius on the track. This can be determined using the following formulae.

Now once the geometry has been decided, a few more things like caster, bump steer and making the geometry into a real-world steering system need to be focused on.

\section{Caster and mechanical trail}

As mentioned before, the steering feel on the car is of utmost importance and the car should also have some self centring effect. However, if it is too much the driver will become uncomfortable.

The total aligning torque of the tires is determined by the mechanical trail and pneumatic trail. The pneumatic trail of the tires can be seen using tire data as it is a property of the tyre. The mechanical trail depends on the caster. The key in setting caster is to see how much camber change is occurring and what mechanical trail is needed.

The mechanical trail should be such that it does not over power pneumatic trail and it should not be too less also so that peak of self aligning torque can be achieved at higher slip angles. The self aligning torque peak is reached much before the Lateral force peak and so a higher peak of SAT will help driver to drive much more near the peak lateral force range and also have a good steering feel.

Looking at the tire data the pneumatic trail is around $30 \mathrm{~mm}$. So a mechanical trail of $40 \mathrm{~mm}$ is chosen to keep the SAT peak higher without making steering force required by the driver too high. A provision can be kept on the wishbone mounts to keep caster adjustable, so that the system can be tested on track.

\section{Mechanical Trail: $10 \mathrm{~mm}$}

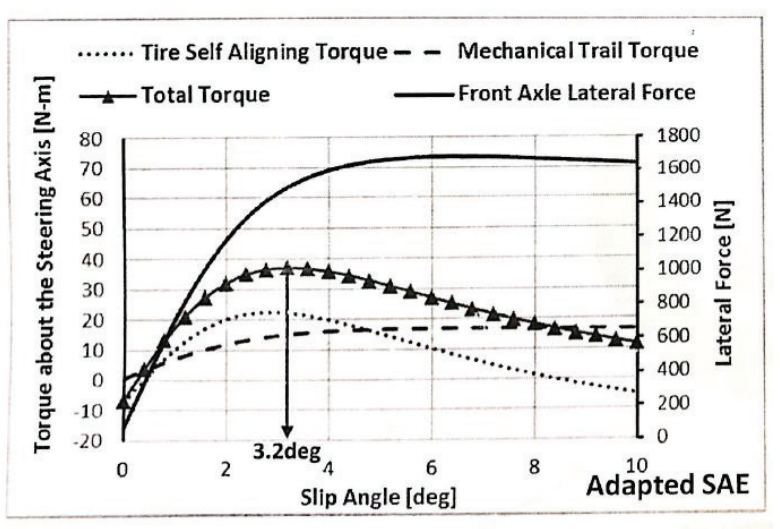

Figure 5. Steering torque vs slip angle (10 $\mathrm{mm}$ Mechanical trail)

\section{Mechanical Trail: $30 \mathrm{~mm}$}

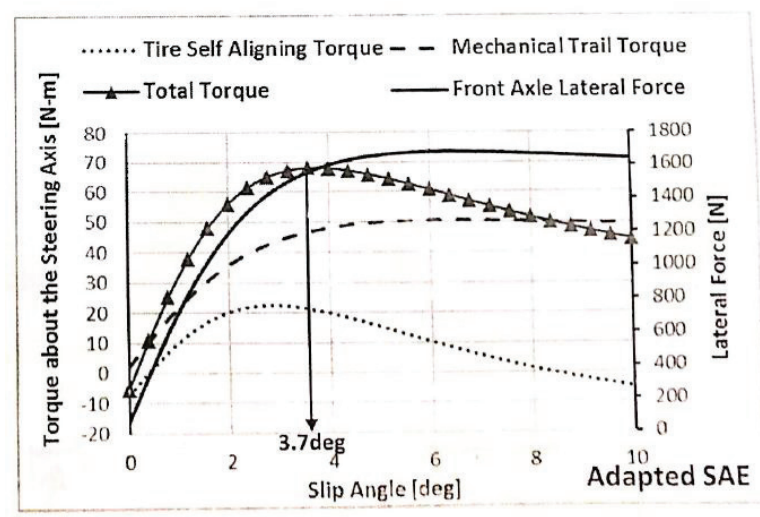

Figure 6. Steering torque vs slip angle (30 $\mathrm{mm}$ Mechanical trail)

\section{Bump steer}

Bump steer is a generally a negative characteristic and should be minimized. The two ways of doing it are first by using the right geometric design and then putting the points in the kinematic software and optimizing it. The geometrical ay to do it is shown below. The position of rack and pinion along the length of the car and the 
outboard toe link point affect bump steer. Toe base should be kept maximum and toe link should be perpendicular to chassis for best compliance related results

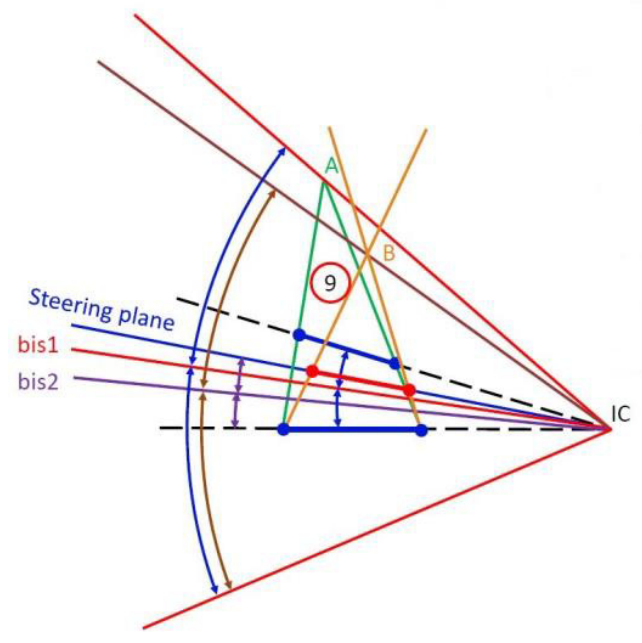

Figure 7. Geometry for minimum bump steer

The best results can be obtained if the toe link is facing the instantaneous centre. The below diagram gives a brief idea about how to go about creating negligible bump steer.

Once the diagram is done and points are known they can be entered into a kinematics software for simulation and further optimized.

\section{Steering rack location}

The location of the steering rack is very important in determining what type of geometry is used. The following table shows what each rack position and the outboard steering points with respect to outboard wishbone pickup points signifies.

Table 1. Effect of various parameters on steering geometry types

\begin{tabular}{|l|c|c|}
\hline $\begin{array}{c}\text { Steering } \\
\text { Rack } \\
\text { Location }\end{array}$ & $\begin{array}{c}\text { Outboard Steering } \\
\text { points with respect } \\
\text { to outer A-arm } \\
\text { points }\end{array}$ & $\begin{array}{c}\text { Type of } \\
\text { steering } \\
\text { Geometry }\end{array}$ \\
\hline Front Steer & Inside & $\begin{array}{c}\text { anti- } \\
\text { Ackerman }\end{array}$ \\
\hline Front Steer & Inline & Parallel \\
\hline Front Steer & Outboard & Ackerman \\
\hline Rear Steer & Inside & Ackerman \\
\hline Rear Steer & Inline & Parallel \\
\hline Rear Steer & Outboard & anti- \\
\hline
\end{tabular}

\section{Roll centre and steering linkages}

As opposed to popular belief the position of the roll centre is affected by the position of the toe link. This is because roll centre is not a 2-D concept but is in fact a 3$\mathrm{D}$ concept. The method is based on the Reuleaux method, which is a geometrical method to locate the instant centre of rotation of a planar motion.

\section{Conclusion}

The focus of this paper is to give an idea on how to use tire data, kinematic software and use of other important parameters to design a competitive steering system which will give highest performance for the set of tyres. Important points like roll centre relation with steering linkage, use of peak lateral force along with the choice of Anti-Ackermann or pro Ackermann have been explained. It covers all important points needed to understand the designing of a steering system for providing peak performance. Trends have been generated to show the significance of change of $\mathrm{RCH}$ with change in steering linkage coordinates.

The influence of other parameters like bump steer, caster and mechanical trail have also been discussed. The significance of tire drag which is not generally given enough mention has also been discussed.

\section{References}

1. www.iaeng.org/publication/WCE2016/WCE2016_pp 1062-1066.pdf

2. Ganesh Mohan, Claude Rouelle, Emmanuel Hugon, OptimumG LLC, Denver, Colorado A New Method to Evaluate Bump Steer and Steering Influence on Kinematic Roll and Pitch Axes for All Independent Suspension Types

3. http://www.optimumg.com/docs/SteeringGeometry.p df

4. William F. Milliken and Douglas L. Milliken, 1995, "Race Car Vehicle Dynamics", Society of Automotive Engineers, Inc.

5. Gaffney, Edmund F., and Anthony R. Salinas. Introduction to Formula SAE® Suspension and Frame Design. No. 971584. SAE Technical Paper, 1997.

6. Robertson, Dennis, and George J. Delagrammatikas. The suspension system of the 2009 cooper union FSAE vehicle: A comprehensive design review. No. 2010-01-0311. SAE Technical Paper, 2010.

7. Gillespie, Thomas D., Fundamentals of Vehicle Dynamics. Society of Automotive Engineers, Inc., Pennsylvania, 1992.

8. Smith, Carroll, Engineer to Win. MBI Publishing Company, Minnesota, 1984.

9. Norton, R. Machine design. Pearson Prentice Hall, Upper Saddle River, NJ, 2006.

10. Heisler, H. (1989), Advanced Vehicle Technology, Arnold, 338 Euston Road, London NW1 3BH

11. Fenton, J. (1980), Vehicle Body Layout and Analysis, Mechanical Engineering Publications Ltd. London 
12. Smith, C. (1978), Tune to Win: The Art and Science of Race Car Development and Tuning, Aero Publishers, Inc. 329 West Aviation Road, Fallbrook, CA 29028

13. Vehicle Dynamics Terminology- SAEJ670, Revise 07-1976.
14. Pashley, T. (2008). Hoe to Build Motorcycle-engined racing cars, UK, Veloce Publishing 